\title{
Detection of Leptospirosis Bacteria in Rodent Urine by Surface Plasmon Resonance Sensor Using Graphene
}

\author{
Surjeet RAIKWAR ${ }^{1}$, Y. K. PRAJAPATI ${ }^{2 *}$, D. K. SRIVASTAVA ${ }^{3}$, \\ J. B. MAURYA ${ }^{4}$, and J. P. SAINI ${ }^{5}$ \\ ${ }^{1,3,4}$ Department of Electronics and Communication Engineering, Bundelkhand Institute of Engineering and Technology, \\ Jhansi 284128, Uttar Pradesh, India \\ ${ }^{2}$ Department of Electronics and Communication Engineering, Motilal Nehru National Institute of Technology Allahabad, \\ Prayagraj 211004, Uttar Pradesh, India \\ ${ }^{5}$ Netaji Subhas University of Technology, Dwarka 110078, New Delhi, India \\ *Corresponding author: Y. K. PRAJAPATI_ E-mail: yogendrapra@gmail.com and yogendrapra@mnnit.ac.in
}

\begin{abstract}
In this paper, a graphene-coated surface plasmon resonance sensor is designed for the examination of Rodent urine which is responsible for Leptospirosis bacteria. Rodent urine is considered as sensing medium. Graphene surface is activated by phosphate-buffered saline solution for better attachment of Leptospirosis bacteria on its surface. Oliguria and Polyuria are the Rodent urine with high and low concentrations of Leptospirosis bacteria, respectively. The transfer matrix method is used for the formulation of reflection intensity of p-polarized light. The reflectance curves for angular interrogation are plotted and the results are obtained in terms of sensitivity, detection accuracy, and quality factor. The significantly high sensitivity and detection accuracy for Oliguria distinguishes it from Polyuria having lower sensitivity.
\end{abstract}

Keywords: Surface plasmon resonance sensor; active metal layer; Leptospirosis bacteria

Citation: Surjeet RAIKWAR, Y. K. PRAJAPATI, D. K. SRIVASTAVA, J. B. MAURYA, and J. P. SAINI, "Detection of Leptospirosis Bacteria in Rodent Urine by Surface Plasmon Resonance Sensor Using Graphene," Photonic Sensors, 2021, 11(3): 305-313.

\section{Introduction}

Leptospirosis is widely spread zoonosis in the world. Leptospirosis caused by spirochetes belongs to Leptospira genus which is sub-classified in pathogenic and saprophytic species (chemoheterotrophic in nature). Leptospira is thin, having long spiral-shaped (screw shape) cell, with length of $6 \mu \mathrm{m}-20 \mu \mathrm{m}$ and diameter of $0.09 \mu \mathrm{m}-$ $3.00 \mu \mathrm{m}$. Leptospira has surface features of both Gram-negative bacteria [1]. Leptospirosis is transmitted to human by either directly infected animal's urine of skunks, raccoons, foxes (often Rodents' urine) or indirectly by contaminated water and soil [2]. This Leptospirosis is responsible for diseases in humans like mild flu, yellow fever, hepatic failure, hemorrhage, and infection in pulmonary track which can lead to death [3]. The developing countries are mostly affected by these diseases; millions cases are reported yearly. Approximately 1.03 million cases out of which 58900 deaths worldwide due to Leptospirosis were reported [4]. Various traditional laboratory diagnoses of Leptospirosis bacteria under the

Received: 27 August 2019 / Revised: 03 April 2020

(C) The Author(s) 2020. This article is published with open access at Springerlink.com

DOI: 10.1007/s13320-020-0587-0

Article type: Regular 
categories; microscopy, serology (antibody and antigen), and molecular were reported by S. N. Ahmed et al. [5]. These traditional methods are slow, non-repeatable, requiring large sample, complex, and having low sensitivities [5]. In contrast, the surface plasmon resonance (SPR) sensor is a label-free detection technique in real-time. The SPR sensor has capability to detect the presence of single biomolecule with high sensitivity. In addition, this technique is fast and repeatable. This SPR sensor has various applications in industries, mines, food safety, medicine investigation, materials synthesis, etc. $[6,7]$. Further, the SPR sensor is also used for the study of interaction between different bio species, e.g., nucleic acid, antibodies, bacteria, and fungi.

The SPR sensor was first configured by $\mathrm{H}$. Reather and E. Kretschmann in 1968 and coined as Kretschmann configuration [8]. In this configuration, a thin film of plasmonic metal like gold is directly deposited on the dielectric, such as glass substrate. Otto's configuration of the SPR sensor, contemporary of Kretschmann configuration, was not popular just because of maintaining a thin air gap between plasmonic metal and a dielectric substrate. Further, in 1983, B. Liedberg et al. [9] utilized this Kretschmann configuration as SPR biosensor for the detection of gas.

Primary plasmonic metals to generate surface plasmons are gold, silver, copper, aluminum, sodium, and indium [10]. Indium is very expensive, sodium is reactive, and silver, copper, and aluminum are much oxidizable. In contrast, gold is the best choice as plasmonic metal. But, as far as the performance of the SPR sensor is concerned, silver has moderate sensitivity with the highest detection accuracy and quality factor, and copper has the highest sensitivity [10]. Hence, silver can be the best plasmonic metal provided that it should be protected from oxidation and corrosion.

Immobilization of antibody/antigen for Leptospirosis on the bare plasmonic metal is not very useful [11]. There should be some material which has higher affinity towards plasmonic metal as well as antibody/antigen for Leptospirosis. Also, plasmonic metals must be protected from oxidation and corrosion. Graphene is chemical inert in nature [12] and can easily be attached on the plasmonic metal, and it can also bind the carbon ring of biomolecule through pi-stacking property [13]. Also, graphene on plasmonic metal has higher sensitivity than bare plasmonic metals $[14,15]$. In addition, graphene improves the confinement, propagation distance, and electrostatic tunability by enhancing the light-matter interaction $[16,17]$. The phosphate buffered saline (PBS) solution will impact the final refractive index increases and help in hydrating the bacteria deposited on the biosensor layer [18].

It is reported that bacteria have higher affinity towards hydrophobic surfaces [19]. In addition, hydrophobic surfaces adsorb the bacteria faster with stronger forces. It is worth noting here that the hydrophobic nature of graphene surface depends upon the production method and oxide related functional groups [20]. However, the pristine graphene surface is a kind of hydrophobic surface. Therefore, it is believed that Leptospirosis bacteria can easily be adsorbed on the graphene surface. Further, this attachment may depend upon the orientation of graphene surface with respect to bacteria [20]. The morphological study shows that Leptospira have unique hook type structure at both ends [1]. Therefore, the highly conformal and flexible encasement of graphene may trap the Leptospirosis bacteria which could play a major role in the selectivity of the proposed sensor. In this paper, the different refractive indices corresponding to different concentrations of Leptospirosis bacteria in the Rodents contaminated urine have been studied. The design parameters, mathematical formulation, and performance parameters are defined in Section 2. Results are discussed in Section 3. The paper ends with a remarkable conclusion and referencing. 


\section{Theory and numerical formulation}

\subsection{Sensor structure and design parameters}

The schematic diagram of the nanomaterial-based SPR sensor structure is presented in Fig. 1. Here, silver is considered as plasmonic metal. Silver is covered with graphene to protect it from oxidation and high affinity towards Leptospirosis. Then PBS is poured on the graphene surface to improve the attachment of Leptospirosis. The sensing medium is Rodent urine kept over the PBS solution. At the operating wavelength $633 \mathrm{~nm}$, the refractive indices of BK-7 prism, silver, Graphene, and PBS solution are 1.5151 [21], $0.15677+3.8045 \mathrm{i}$ [22], 3+1.1491 06i [21], and 1.334 [22], respectively. The refractive index (RI) of sensing medium $\left(n_{s}\right)$ is considered to vary from 1.33 (pure water) to polyuria (water volume more than Rodent urine), and oliguria (water volume less the Rodent urine). Ten samples of Polyuria and nine samples of Oliguria whose refractive indices were calculated by Y. F. Kitagawa et al. [23] are considered for sensing medium and listed in Table 1. Each of these samples for Polyuria and Oliguria is collected from different rodents. The thicknesses of silver, Graphene, PBS solution are $52 \mathrm{~nm}, 0.34 \mathrm{~nm}$, and $3 \mathrm{~nm}$, respectively.

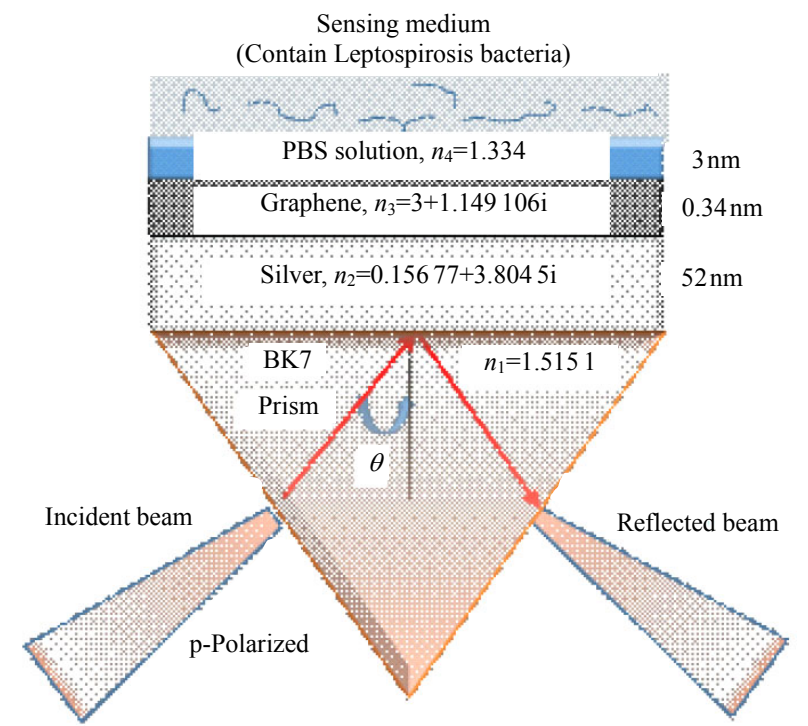

Fig. 1 Kretschmann configuration-based prism coupled SPR sensor.

\subsection{Principle of operation}

To obtain the condition of resonance, a $633 \mathrm{~nm}$ wavelength of $\mathrm{He}-\mathrm{Ne}$ p-polarized laser source is used to focus on the lateral plane of a glass prism. After that light is directed on the thin deposited silver film. By the principle of attenuated total reflection (ATR), evanescent field excites the plasmons at the silver surface. After achieving the resonance condition, a surface plasmon wave (SPW) starts to propagate at the metal/dielectric interface. This resonance condition can be achieved by matching the wave vector of incident light with that of the SPW. The wave vector of incident can be varied by varying the incident angle [6]. The angle at which resonance condition is achieved is known as resonance angle. The resonance condition is always obtained at angle greater than critical angle $\left\{\theta_{c}=\sin ^{-1}\left(n_{c} / n_{\text {prism }}\right)\right\}$, i.e., $\theta_{\text {res }}>\theta_{c}$. This SPW is highly sensitive to the change in refractive index near the interface. Hence, any change in refractive index due to absorption or adsorption disturbs the resonance condition which can be again achieved by changing the incident angle. The shift in resonance angle is an important parameter to define the sensitivity of the sensor. This change is captured by the SPR setup containing a photo-detector and a lock-in amplifier to plot the SPR curve known as reflectance curve.

\subsection{Numerical modeling of reflectivity (R)}

The reflectivity is calculated by the $N$-layer matrix method. We consider $d_{k}$ as a thickness of layer along $z$-plane. The materials used for sensor have dielectric constant and RI, which are represented as $\varepsilon_{k}$ and $n_{k}$, respectively. By using the boundary condition, the tangential fields along first boundary, $Z=Z_{1}=0$ are presented in terms of the tangential fields of the last boundary $Z=Z_{N-1}$ as follows:

$$
\left[\begin{array}{l}
V_{1} \\
W_{1}
\end{array}\right]=\mathbf{X}_{i j}\left[\begin{array}{l}
V_{N-1} \\
W_{N-1}
\end{array}\right]
$$


Table 1 Performance parameters for different rats of Polyuria and Oliguria category presented by Y. F. Kitagawa et al. [23].

\begin{tabular}{|c|c|c|c|c|c|c|c|c|c|c|c|}
\hline $\begin{array}{l}\text { Rat } \\
\text { No. }\end{array}$ & $\begin{array}{l}\text { Water } \\
(\mathrm{ml})\end{array}$ & $\begin{array}{l}\text { Urine } \\
(\mathrm{ml})\end{array}$ & $\begin{array}{l}\text { Refractive } \\
\text { index (RI) }\end{array}$ & $\begin{array}{l}\text { Resonance } \\
\text { angle }\left(\theta_{\text {res }}\right)\end{array}$ & $R_{\min }$ & $F W H M$ & $\delta n_{\mathrm{c}}$ & $\Delta \theta_{\mathrm{res}}$ & $\begin{array}{c}\text { Sensitivity } \\
\left(S_{n}\right)\end{array}$ & $\begin{array}{c}\text { Detection } \\
\text { accuracy }\left(D_{a}\right)\end{array}$ & $\begin{array}{c}\text { Quality factor } \\
\qquad\left(Q_{F}\right)\end{array}$ \\
\hline \multicolumn{12}{|c|}{ Pure water } \\
\hline- & - & - & 1.33 & 69.5052 & 0.031 & 3.0791 & - & - & - & - & - \\
\hline \multicolumn{12}{|c|}{ Polyuria } \\
\hline 1 & 164 & 136 & 1.3337 & 69.9819 & 0.0316 & 3.1507 & 0.0037 & 0.4767 & 128.8378 & 0.1513 & 40.8918 \\
\hline 2 & 126 & 104 & 1.3341 & 70.0340 & 0.0317 & 3.1581 & 0.0041 & 0.5288 & 128.9756 & 0.1674 & 40.8396 \\
\hline 3 & 114 & 102 & 1.3342 & 70.0472 & 0.0318 & 3.1604 & 0.0042 & 0.5420 & 129.0476 & 0.1715 & 40.8327 \\
\hline 5 & 64 & 57 & 1.3344 & 70.0736 & 0.0318 & 3.1644 & 0.0044 & 0.5684 & 129.1818 & 0.1796 & 40.8235 \\
\hline 4 & 88 & 63 & 1.3345 & 70.0868 & 0.0318 & 3.1662 & 0.0045 & 0.5816 & 129.2444 & 0.1836 & 40.8201 \\
\hline 6 & 62 & 38 & 1.3351 & 70.1652 & 0.0319 & 3.1776 & 0.0051 & 0.6600 & 129.4118 & 0.2077 & 40.7263 \\
\hline 7 & 50 & 41 & 1.3353 & 70.1916 & 0.0320 & 3.1822 & 0.0053 & 0.6864 & 129.5094 & 0.2157 & 40.6981 \\
\hline 10 & 38 & 32 & 1.3355 & 70.2180 & 0.0320 & 3.1862 & 0.0055 & 0.7128 & 129.6000 & 0.2237 & 40.6754 \\
\hline 8 & 40 & 19 & 1.3365 & 70.3503 & 0.0322 & 3.2057 & 0.0065 & 0.8451 & 130.0154 & 0.2636 & 40.5576 \\
\hline 9 & 40 & 21 & 1.3375 & 70.4832 & 0.0325 & 3.2263 & 0.0075 & 0.9780 & 130.4000 & 0.3031 & 40.4178 \\
\hline \multicolumn{12}{|c|}{ Oliguria } \\
\hline 107 & 8 & 11 & 1.3392 & 70.7113 & 0.0329 & 3.2613 & 0.0092 & 1.2061 & 131.0978 & 0.3690 & 40.1980 \\
\hline 108 & 10 & 14 & 1.3401 & 70.8327 & 0.0331 & 3.2802 & 0.0101 & 1.3275 & 131.4356 & 0.4047 & 40.0694 \\
\hline 109 & 10 & 12 & 1.3406 & 70.9009 & 0.0333 & 3.2899 & 0.0106 & 1.3957 & 131.6698 & 0.4242 & 40.0224 \\
\hline 105 & 6 & 13 & 1.3409 & 70.9416 & 0.0334 & 3.2968 & 0.0109 & 1.4364 & 131.7798 & 0.4357 & 39.9720 \\
\hline 103 & 4 & 9 & 1.3410 & 70.9554 & 0.0334 & 3.2991 & 0.0110 & 1.4502 & 131.8364 & 0.4395 & 39.9613 \\
\hline 104 & 6 & 4 & 1.3423 & 71.1335 & 0.0338 & 3.3260 & 0.0123 & 1.6283 & 132.3821 & 0.4895 & 39.8022 \\
\hline 102 & 4 & 6 & 1.3445 & 71.4389 & 0.0344 & 3.3730 & 0.0145 & 1.9337 & 133.3586 & 0.5732 & 39.5371 \\
\hline 106 & 8 & 8 & 1.3449 & 71.4951 & 0.0346 & 3.3822 & 0.0149 & 1.9899 & 133.5503 & 0.5883 & 39.4862 \\
\hline 101 & 2 & 4 & 1.3470 & 71.7919 & 0.0353 & 3.4286 & 0.0170 & 2.2867 & 134.5118 & 0.6669 & 39.2323 \\
\hline
\end{tabular}

where $V_{1}$ and $W_{1}$ represent the tangential component of electric and magnetic fields at the first boundary, respectively, and $V_{N-1}$ and $W_{N-1}$ are the corresponding fields at the $(N-1)$ th boundary. $X_{i j}$ denotes the characteristic transfer matrix that can be obtained through the following $2 \times 2$ matrix of the complete sensor structure excited by p-polarized light:

$$
\mathbf{X}_{i j}=\left(\prod_{k=2}^{N-1} \mathbf{X}_{k}\right)_{i j}=\left[\begin{array}{ll}
X_{11} & X_{12} \\
X_{21} & X_{22}
\end{array}\right]
$$

with

$$
\mathbf{X}_{k}=\left[\begin{array}{cc}
\cos \beta_{k} & \left(-\mathrm{i} \sin \beta_{k}\right) / q_{k} \\
-\mathrm{i} q_{k} \sin \beta_{k} & \cos \beta_{k}
\end{array}\right]
$$

where

$$
q_{k}=\frac{\left(\varepsilon_{k}-n_{1}^{2} \sin ^{2} \theta_{1}\right)^{1 / 2}}{\varepsilon_{k}}
$$

$$
\beta_{k}=\frac{2 \pi d_{k}}{\lambda}\left(\varepsilon_{k}-n_{1}^{2} \sin ^{2} \theta_{1}\right)^{1 / 2}
$$

where $\beta_{k}$ and $q_{k}$ represent the phase constant and transfer refractive index of the $k$ th layer, respectively. $\theta_{1}$ denotes the incident angle with respect to the normal to the first layer and $n_{1}$ denotes the RI of the first layer. By using the above mathematical equation, the reflection coefficient $\left(r_{p}\right)$ is calculated as

$$
r_{p}=\frac{\left(X_{11}+X_{12} q_{N}\right) q_{1}-\left(X_{21}+X_{22} q_{N}\right)}{\left(X_{11}+X_{12} q_{N}\right) q_{1}+\left(X_{21}+X_{22} q_{N}\right)}
$$

The magnitude of the reflection intensity $\left(R_{p}\right)$ of the defined multilayer configuration is given as

$$
R_{p}=\left|r_{p}\right|^{2}
$$




\subsection{Performance parameters calculated for the proposed SPR sensor}

The performance of the SPR sensor can be measured by three parameters viz sensitivity, detection accuracy, and quality factor. Sensitivity $S_{n}$ is the ratio of a shift in resonance angle to $\delta \theta_{\mathrm{es}}$ corresponding to the change in refractive index of the sensing medium $\delta n_{s}$

$$
S_{n}=\frac{\delta \theta_{\text {res }}}{\delta n_{s}} \quad \text { (unit: deg. /RIU) }
$$

Detection accuracy (DA), also known as resolution, defines the exactness of measured signal. It depends upon the accuracy of measurement of resonance angle which is limited by system noise [24]. It can be formulated as the ratio of a shift in resonance angle to the beam width of SPR curve. This beam width can be measured as full width at half maxima (FWHM) [25]. Narrower FWHM results to a higher detection accuracy which provides exact calculation of resonance angle.

$$
D A=\frac{\delta \theta_{\text {res }}}{F W H M} \text { (unit less quantity) }
$$

Quality factor $\left(Q_{F}\right)$ is the ratio of sensitivity to FWHM of SPR sensor.

$$
Q_{F}=\frac{S_{n}}{F W H M} \text { (unit: } \mathrm{RIU}^{-1} \text { ) }
$$

To obtain high performance of any sensors, all of the three parameters, i.e., $S_{n}, D A$, and $Q_{F}$ should be as high as possible.

\section{Results and discussion}

Leptospirosis bacteria presented in Rodent urine can be immobilized on the graphene surface by the enhanced attachment through PBS solution. These immobilized Leptospirosis change the molar concentration of solution (PBS) which again change the RI of sensing medium. The change in RI will affect the propagation constant of surface plasmons waves resulting in a disturbance in the resonance condition, which can be further achieved at different angles of incidence. Thus, to detect the presence of Leptospirosis bacteria, angular interrogation is adapted.

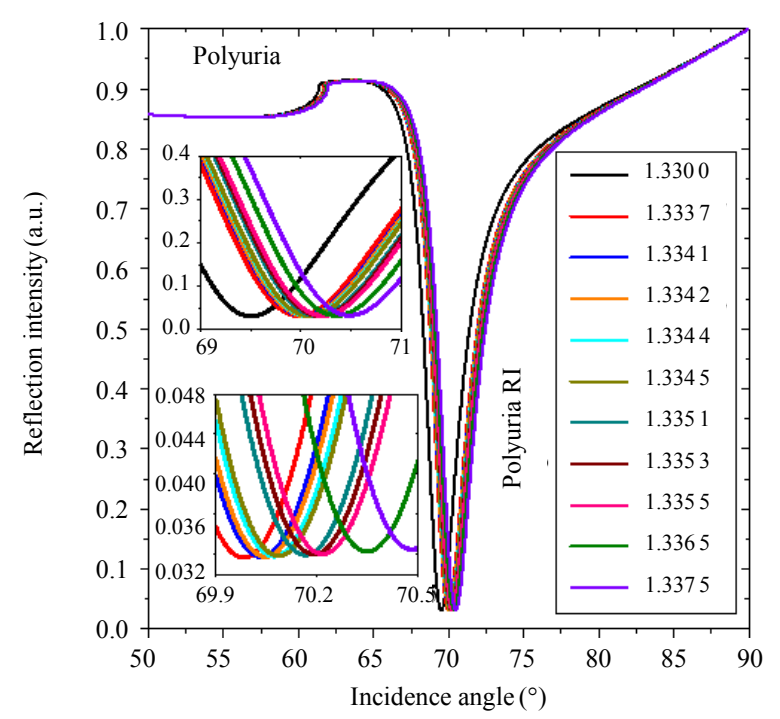

(a)

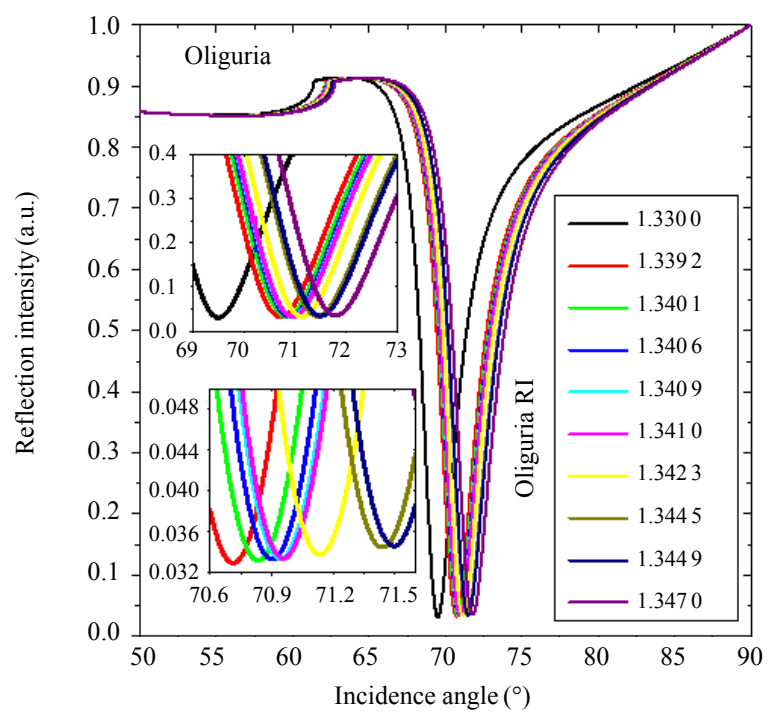

(b)

Fig. 2 Reflection intensity vs incident angle, i.e., SPR curves are plotted for different sensing medium refractive indices with respect to pure water: (a) Polyuria and (b) Oliguria.

The reflection intensity is varied by the incident angle at different refractive indices under Polyuria and Oliguria in Figs.2(a) and 2(b), respectively. The obtained reflectance curves are of desired pattern and shape. The critical angle corresponding to pure water can be calculated as $\theta_{c}=\sin ^{-1}\left(n_{c} / n_{\text {prism }}\right)$, i.e., $61.38^{\circ}$. It can be easily observed from these figures and Table 1 that the resonance angles of all the reflectance curves are higher than the critical angle, i.e., $\theta_{\mathrm{res}}>\theta_{c}$, which is primary requirement of surface plasmon resonance. It can also be observed that the reflection intensity is minimum at these resonance 
angles, which justifies that the maximum intensity is utilized to generate the surface plasmons [26]. The refractive indices of different Rodent urine samples under Polyuria and Oliguria are arranged in an increasing order in Table 1. The resonance angle increases with an increase in the refractive index of Rodent urine. All the resonance shifts are measured with respect to the resonance angle of pure water. Higher change in concentration near the graphene surface with respect to pure water due to the adsorption of Leptospirosis bacteria changes the resonance condition with higher amount. This high change in resonance condition needs a significant change in an incident angle to achieve the resonance condition. This can be easily seen from Figs. 2(a) and 2(b) and Table 1 that as the refractive index of Rodent urine increases, the shift in the resonance angle increases. Therefore, higher concentration of Rodent urine in Oliguria justifies the higher resonance angle shift of Oliguria with respect to Polyuria (See Table 1). As the refractive index increases near the graphene surface, the surface plasmons starts to damp. This damping increases with a further increase in the refractive index [27]. This plasmon damping is responsible for broadening and shallowing of reflectance curves which can be easily observed from Figs. 2(a) and 2(b). The $F W H M$ and $R_{\min }$ increase with a refractive index (see Table 1) just because of the broadening and shallowing of reflectance curves due to plasmon damping. From Table 1 , it can be noted that $R_{\min }$ varies from 0.0316 to 0.0325 for Polyuria and from 0.0329 to 0.0353 for Oliguria, and $F W H M$ varies from 3.1507 to 3.2263 for Polyuria and from 3.2613 to 3.4286 for Oliguria. It can be calculated that the changes in $R_{\min }$ are 0.0009 and 0.0024 for Polyuria and Oliguria, respectively, which are very low.

Further, the changes in FWHM are 0.0756 and 0.1673 for Polyuria and Oliguria, respectively, which are not negligible. Hence, the $R_{\min }$ is not significantly affected by change in refractive index, whereas $F W H M$ is affected. Although an increase in $F W H M$ is significant, these reflectance curves are considered for the measurement of resonance angle.

Based on reflectance curves obtained at different refractive indices under Polyuria and Oliguria in Figs. 2(a) and 2(b), performance parameters, i.e., sensitivity, detection accuracy, and quality factors, are plotted in Figs. 3(a) - 3(c), respectively. The vertical dashed black line in Figs. 3(a) -3(c) defines the boundary between refractive indices of Polyuria and Oliguria. Leftward to this vertical line, the refractive index corresponds to Polyuria whereas rightward is Oliguria. It can be seen from Table 1 that the shift in the resonance angle increases with the refractive index of Rodent urine, and according to (8), the sensitivity is directly proportional to the change in the resonance angle, hence the sensitivity increases monotonically with an increase in the refractive index of Rodent urine which can be clearly observed from Fig. 3(a) and Table 1. It can be seen from Table 1 that the sensitivity corresponding to Oliguria varies from 131.0978 (deg./RIU) to 134.5118 (deg./RIU) whereas for Polyuria varies from 128.8378 (deg./RIU) to 130.40 (deg./RIU). Hence, it can be concluded that the sensitivity for Oliguria is much higher than that for Polyuria. It can be easily calculated from Table-1 that the change in $\delta \theta_{\text {res }}$ is higher than that in $F W H M$, corresponding to any change in the refractive index of Rodent urine $\delta n_{s}$. Thus, according to (9), the detection accuracy monotonically increases with the refractive index of Rodent urine which can be observed from Fig. 3(b) and Table 1. It can be seen from Table 1 that the detection accuracy corresponding to Oliguria varies from 0.36982 to 0.5883 whereas for Polyuria varies from 0.1513 to 0.3031 . Hence, it can be concluded that the detection accuracy for Oliguria is much higher than that for Polyuria. From (8)-(10), the quality factor can be defined as the ratio of detection accuracy to the corresponding change in refractive index of Rodent urine. Also, from Table 1 it can be calculated that change in detection accuracy is lower than that in $\delta n_{s}$. Therefore, the quality factor decreases monotonically with the refractive index of Rodent urine. It can be seen from Table 1 that the 
quality factor corresponding to Oliguria varies from 40.198 to 39.2323 whereas for Polyuria varies from 40.8918 to 40.4178 . Hence, it can be concluded that the quality factor for Oliguria is lower than that for Polyuria. Further, it has been already shown in the previous articles of our research group that the sensitivity, detection accuracy, and quality factor degrade with the increasing chemical potential of graphene and are almost constant with the temperature $[28,29]$.

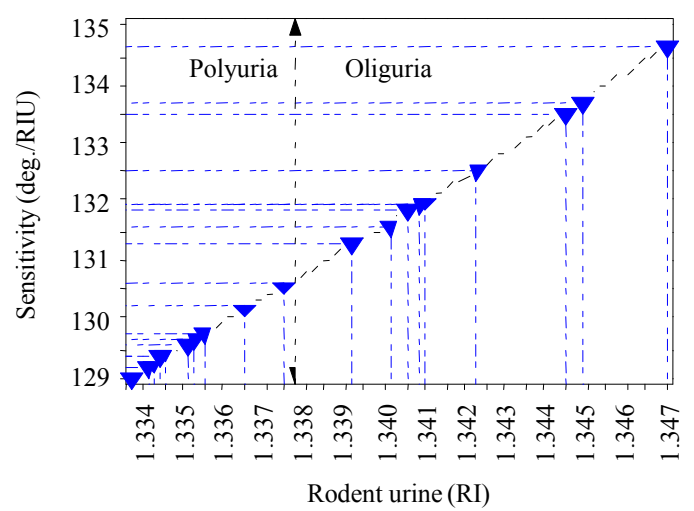

(a)

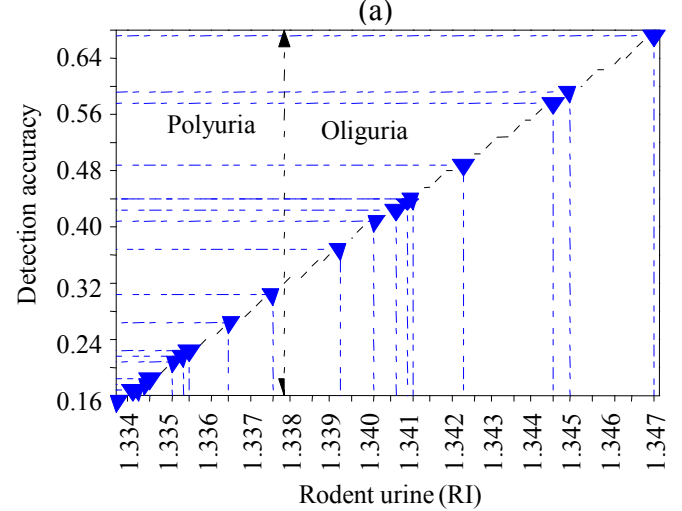

(b)

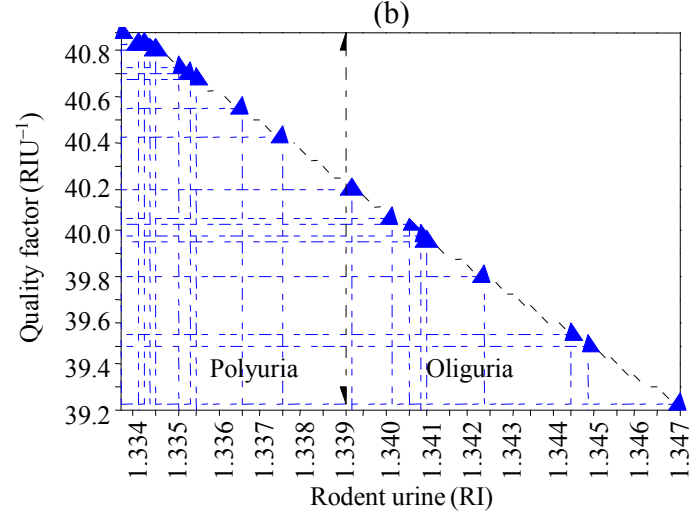

(c)

Fig. 3 Variation of performance parameters with respect to different sensing medium refractive indices under pure water, Polyuria, and Oliguria: (a) sensitivity, (b) detection accuracy, and (c) quality factor.

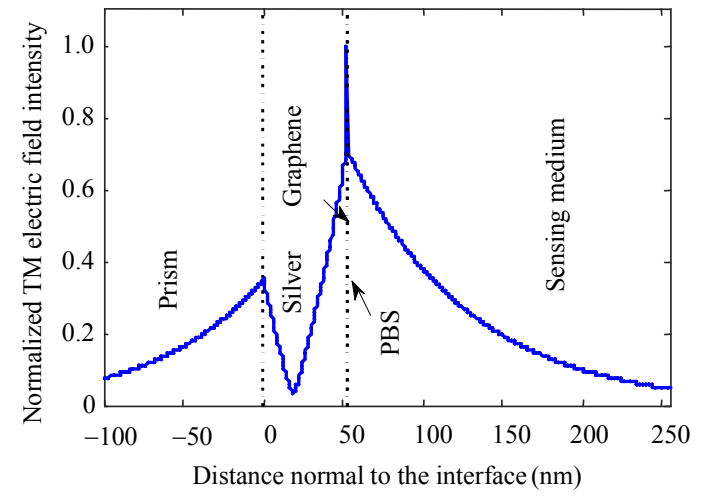

Fig. 4 Transfer magnetic (TM) electric field intensity as the function of distance normal to interface of a different layer.

The distribution of normalized electric field in different layers of proposed sensor is obtained by plotting TM electric field at 1.33 refractive index of sensing medium. Here, the electric field in a particular layer is normalized with respect to the maximum electric field in that layer. Increasing the electric field signifies the excitation of surface plasmons [26]. The resonance condition is achieved at the maximum electric field at silver/sensing medium interface. Graphene decreases the electric field inside it due to which surface plasmons gets damped. It can be seen from Fig. 4 that the electric field decreases exponentially in the sensing medium. The penetration depth in the sensing medium can be calculated as distance covered in the sensing medium from the maximum electrical field to the 1/e of the maximum electric field. The obtained penetration depth is $76 \mathrm{~nm}$. High penetration depth signifies the high sensing volume for interaction with Leptospirosis bacteria or any other antigen [30].

\section{Conclusions}

The proposed Graphene-based SPR sensor is analyzed theoretically and numerically for the detection of Leptospirosis bacteria in the Rodent urine. The sensitivity and detection accuracy increase whereas the quality factor decreases with an increase in the refractive index of Rodent urine just because of an increase in Leptospirosis bacteria. It is 
believed that the proposed sensor can be utilized for the early detection of diseases related to Leptospirosis bacteria with high sensitivity and accuracy.

\section{Acknowledgment}

Surjeet Raikwar is thankful to Dr. Abdul Kalam Technical University, Lucknow (India) for providing Research Grant Homi Bhabha Teaching Assistant Fellowship.

Open Access This article is distributed under the terms of the Creative Commons Attribution 4.0 International License (http://creativecommons.org/licenses/by/4.0/), which permits unrestricted use, distribution, and reproduction in any medium, provided you give appropriate credit to the original author(s) and the source, provide a link to the Creative Commons license, and indicate if changes were made.

\section{References}

[1] P. N. Levett, "Leptospirosis," Clinical Microbiology Reviews, 2001, 14(2): 296-326.

[2] A. Packchanian, "Susceptibility and resistance of certain species of American deer mice, genus Peromyscus, and other rodents to Leptospira Icterohaemorrhagiae," Public Health Reports (1896-1970), 1940, 55(31): 1389-1402.

[3] S. Faine, B. Adler, C. Bolin, and P. Perolat, Leptospira and leptospirosis. Melbourne, Australia: MediSci, 1999

[4] F. Costa, J. E. Hagan, J. Calcagno, M. Kane, P. Torgerson, M. S. Martinez-Silveira, et al., "Global morbidity and mortality of leptospirosis: a systematic review," Plos Neglected Tropical Diseases, 2015, 9(9): e0003898.

[5] S. N. Ahmed, S. Shah, and F. M. H. Ahmad, "Laboratory diagnosis of leptospirosis," Journal of Postgraduate Medicine, 2005, 51(3): 195.

[6] J. Homola, "Present and future of surface plasmon resonance biosensors," Analytical and Bioanalytical Chemistry, 2003, 377(3): 528-539.

[7] J. B. Maurya and Y. K. Prajapati, "Influence of dielectric coating on performance of surface plasmon resonance sensor," Plasmonics, 2017, 12(4): 1121-1130.

[8] E. Kretschmann and H. Raether, "Radiative decay of non radiative surface plasmons excited by light," Zeitschrift für Naturforschung A, 1968, 23(12): 2135-2136.
[9] B. Liedberg, C. Nylander, and I. Lundstrom, "Surface plasmon resonance for gas detection and biosensing," Sensors and Actuators, 1983, 4: 299-304.

[10] J. B. Maurya and Y. K. Prajapati, "A comparative study of different metal and prism in the surface plasmon resonance biosensor having $\mathrm{MoS}_{2}$-graphene," Optical and Quantum Electronics, 2016, 48(5): 280.

[11] L. Wu, H. S. Chu, W. S. Koh, and E. P. Li, "Highly sensitive graphene biosensors based on surface plasmon resonance," Optics Express, 2010, 18(14): 14395-14400.

[12] D. G. Papageorgiou, I. A. Kinloch, and R. J. Young, "Mechanical properties of graphene and graphene-based nanocomposites," Progress in Materials Science, 2017, 90: 75-127.

[13] G. B. McGaughey, M. Georgia, and A. K. Rappe, " $\pi$-stacking interactions alive and well in proteins," Journal of Biological Chemistry, 1998, 273(25): 15458-15463.

[14] B. Song, D. Li, W. Qi, M. Elstner, C. Fan, and H. Fang, "Graphene on Au (111): a highly conductive material with excellent adsorption properties for high-resolution bio/nanodetection and identification," ChemPhysChem, 2010, 11(3): 585-589.

[15] A. Upadhyay, Y. K. Prajapati, and R. Tripathi, "Analytical study of planar waveguide sensor with a metamaterial guiding layer," Photonic Sensors, 2017, 7(4): 377-384.

[16] S. X. Xia, X. Zhai, Y. Huang, J. Q. Liu, L. L. Wang, and S. C. Wen, "Graphene surface plasmons with dielectric metasurface," Journal of Lightwave Technology, 2017, 35(20): 4553-4558.

[17] S. X. Xia, X. Zhai, L. L. Wang, and S. C. Wen, "Plasmonically induced transparency in double-layered graphene nanoribbons," Photonics Research, 2018, 6(7): 692-702.

[18] L. Dieguez, N. Darwish, M. Mir, E. Martinez, M. Moreno, and J. Samitier, "Effect of the refractive index of buffer solutions in evanescent optical biosensors," Sensor Letters, 2009, 7(5): 851-855.

[19] R. J. Doyle and M. Rosenberg, Microbial cell surface hydrophobicity. Washington: American Society for Microbiology, 1990.

[20] S. M. Notley, R. J. Crawford, and E. P. Ivanova, "Bacterial interaction with graphene particles and surfaces," Electoral Studies, 2013, 25(2): 351-368.

[21] R. Kumar, A. S. Kushwaha, M. Srivastava, H. Mishra, and S. K. Srivastava, "Enhancement in sensitivity of graphene-based zinc oxide assisted bimetallic surface plasmon resonance (SPR) biosensor," Applied Physics A, 2018, 124(3): 235.

[22] S. Pal, A. Verma, S. Raikwar, Y. K. Prajapati, and J. P. Saini, "Detection of DNA hybridization using graphene-coated black phosphorus surface plasmon 
resonance sensor," Applied Physics A, 2018, 124(5): 394.

[23] Y. F. Kitagawa, T. Takahashi, and H. Hayashi, "Relationship between the refractive index and specific gravity of the rat urine," Experimental Animals, 1981, 30(3): 307-311.

[24] P. K. Maharana, R. Jha, and S. Palei, "Sensitivity enhancement by air mediated graphene multilayer based surface plasmon resonance biosensor for near infrared," Sensors and Actuators B: Chemical, 2014, 190: 494-501.

[25] A. K. Sharma, R. Jha, and B. D. Gupta, "Fiber-optic sensors based on surface plasmon resonance: a comprehensive review," IEEE Sensors Journal, 2007, 7(8): 1118-1129.

[26] A. Shalabney and I. Abdulhalim, "Electromagnetic fields distribution in multilayer thin film structures and the origin of sensitivity enhancement in surface plasmon resonance sensors," Sensors and Actuators A: Physical, 2010, 159(1): 24-32.

[27] I. Pockrand, "Surface plasma oscillations at silver surfaces with thin transparent and absorbing coatings," Surface Science, 1978, 72(3): 577-588.

[28] A. Verma, A. Prakash, and R. Tripathi. "Sensitivity enhancement of surface plasmon resonance biosensor using graphene and air gap," Optics Communications, 2015, 357: 106-112.

[29] S. Pal, Y. K. Prajapati, and J. P. Saini, "Influence of graphene's chemical potential on SPR biosensor using $\mathrm{ZnO}$ for DNA hybridization," Optical Review, 2020, 27(1): 57-64.

[30] J. B. Maurya, Y. K. Prajapati, V. Singh, and J. P. Saini, "Sensitivity enhancement of surface plasmon resonance sensor based on graphene- $\mathrm{MoS}_{2}$ hybrid structure with $\mathrm{TiO}_{2}-\mathrm{SiO}_{2}$ composite layer," Applied Physics A, 2015, 121(2): 525-533. 